\title{
Lightning driven inner radiation belt energy deposition into the atmosphere: regional and global estimates
}

\author{
C. J. Rodger ${ }^{1}$, M. A. Clilverd ${ }^{2}$, N. R. Thomson ${ }^{1}$, D. Nunn ${ }^{3}$, and J. Lichtenberger ${ }^{4}$ \\ ${ }^{1}$ Department of Physics, University of Otago, Dunedin, New Zealand \\ ${ }^{2}$ Physical Sciences Division, British Antarctic Survey, Cambridge, UK \\ ${ }^{3}$ School of Electronics and Computer Science, Southampton University, Southampton, UK \\ ${ }^{4}$ Space Research Group, Eötvös University, Budapest, Hungary
}

Received: 20 April 2005 - Revised: 3 October 2005 - Accepted: 17 October 2005 - Published: 21 December 2005

\begin{abstract}
In this study we examine energetic electron precipitation fluxes driven by lightning, in order to determine the global distribution of energy deposited into the middle atmosphere. Previous studies using lightning-driven precipitation burst rates have estimated losses from the inner radiation belts. In order to confirm the reliability of those rates and the validity of the conclusions drawn from those studies, we have analyzed New Zealand data to test our global understanding of troposphere to magnetosphere coupling. We examine about $10000 \mathrm{~h}$ of AbsPAL recordings made from 17 April 2003 through to 26 June 2004, and analyze subionospheric very-low frequency (VLF) perturbations observed on transmissions from VLF transmitters in Hawaii (NPM) and western Australia (NWC). These observations are compared with those previously reported from the Antarctic Peninsula. The perturbation rates observed in the New Zealand data are consistent with those predicted from the global distribution of the lightning sources, once the different experimental configurations are taken into account. Using lightning current distributions rather than VLF perturbation observations we revise previous estimates of typical precipitation bursts at $L \sim 2.3$ to a mean precipitation energy flux of $\sim 1 \times 10^{-3} \mathrm{ergs}^{-2} \mathrm{~s}^{-1}$. The precipitation of energetic electrons by these bursts in the range $L=1.9-3.5$ will lead to a mean rate of energy deposited into the atmosphere of $3 \times 10^{-4} \mathrm{ergs} \mathrm{cm}^{-2} \mathrm{~min}^{-1}$, spatially varying from a low of zero above some ocean regions to highs of $\sim 3$ $6 \times 10^{-3} \mathrm{ergs} \mathrm{cm}^{-2} \mathrm{~min}^{-1}$ above North America and its conjugate region.
\end{abstract}

Keywords. Magnetospheric physics (Energetic particles; Precipitating; Magnetosphere-ionosphere interactions) Ionosphere (Active experiments)

Correspondence to: C. J. Rodger

(crodger@physics.otago.ac.nz)

\section{Introduction}

The behaviour of high energy electrons trapped in the Earth's Van Allen radiation belts has been extensively studied, through both experimental and theoretical techniques. During quiet times, energetic radiation belt electrons are distributed into two belts divided by the "electron slot" at $L \sim 2.5$, around which there is relatively low energetic electron flux. In the more than four decades since the discovery of the belts (Van Allen, 1997), it has proved difficult to confirm the principal source and loss mechanisms that control radiation belt particles (Walt, 1996). For some electron energy ranges Whistler-induced Electron Precipitation (WEP) is a significant inner radiation belt loss process (e.g. Dungey, 1963; Rodger et al., 2003), acting as one of the drivers by which whistler mode waves (e.g. plasmaspheric hiss, lightning-generated whistlers) cause pitch angle scattering.

Whistler-induced electron precipitation from the Van Allen radiation belts occurs as a result of coupling between the troposphere and the magnetosphere. The energetic electron precipitation arises from lightning produced whistlers (Storey, 1953) interacting with cyclotron resonant radiation belt electrons near the equatorial zone (Tsurutani and Lakhina, 1997). Pitch angle scattering of energetic radiation belt electrons (Kennel and Petschek, 1966) by whistler mode waves drives some resonant electrons into the bounce loss cone, resulting in their precipitation into the atmosphere (Rycroft, 1973). An important parameter for determining the overall importance of WEP to radiation belt losses is the magnitude of a "typical" WEP event. This may be calculated from theoretical studies (e.g. Abel and Thorne, 1998) or inferred from experimental observations, such as in-situ measurements of WEP events (Voss et al., 1998). A different approach has been to use experimental observations to characterize typical WEP magnitudes. Combining reports of satellite WEP observations with ground based whistler measurements, Rodger et al. (2003) concluded that the typical WEP 
mean precipitation energy flux was $2 \times 10^{-3} \mathrm{ergs} \mathrm{cm}^{-2} \mathrm{~s}^{-1}$, and that in some electron energy ranges WEP may be the most significant inner radiation belt loss mechanism.

One complementary technique to study WEP makes use of long range remote sensing of very low frequency (VLF) waves propagating inside the waveguide bounded by the lower ionosphere and the Earth's surface. Significant variations in the received amplitude and/or phase of fixed frequency VLF transmissions arise from changes in the lower ionosphere. Further discussion on the use of subionospheric VLF propagation as a remote sensing probe can be found in recent review articles (e.g. Barr et al., 2000; Rodger, 2003). WEP leads to localized ionospheric modifications produced by secondary ionisation just below the $D$-region of the ionosphere, which are observed as "Trimpi" perturbations in subionospheric VLF transmissions (Helliwell et al., 1973). These perturbations begin with a relatively fast $(\sim 1 \mathrm{~s})$ change in the received amplitude and/or phase, followed by a slower relaxation $(<100 \mathrm{~s})$ back to the unperturbed signal level due to the recombination of the additional ionisation. Trimpi perturbations permit observers to study WEP fluxes and the chemistry of the nighttime lower ionosphere (e.g. Pasko and Inan, 1994), from locations remote from the actual precipitation region.

Recent studies into the magnitude and time decay of Trimpi perturbations observed at Faraday, Antarctica, have clarified the relationships between WEP mean precipitation energy flux, WEP energy spectra, and VLF perturbation scattering of WEP produced modifications to the ionosphere (Rodger et al., 2004b). Building on this modelling, the dependence of WEP precipitation fluxes on the strength of the associated lightning's return stroke peak current has been identified (Clilverd et al., 2004). In previous studies it has been shown that lightning return stroke current strengths have an approximately linear relationship with the received whistler field strength, and whistler strengths with the size of Trimpi perturbations (Carpenter and LaBelle, 1982; Carpenter and Orville, 1989), from which we would expect a relation between Trimpi scatter amplitude and lightning currents.

Clilverd et al. (2004) considered four study days during which a high proportion of the lightning activity occurring near the east coast of $\mathrm{N}$. America produced observable Trimpi effects on VLF transmitter signals propagating in the region of the Antarctic Peninsula $(\sim L=2-2.5)$. The dependence of the scattered field amplitude on the return stroke peak current of the lightning discharge suggests that during these events diffusion conditions are occurring near the loss cone of the precipitating radiation belt particles due to strong whistler wave fields, probably caused by ducted signals. Three of the four study days showed a high degree of consistency between the levels of lightning return stroke peak current required to produce any given perturbation scatter amplitude value, providing a link between the lightning currents and WEP energy flux (Clilverd et al., 2004). On the remaining day, the Trimpi signatures observed were 6-7 dB greater for a given lightning intensity than on the other study days, consistent with significantly harder radiation belt precipitation spectra (Rodger et al., 2004b), probably caused by post-geomagnetic storm acceleration processes of radiation belt electrons (Meredith et al., 2002).

Investigating the coupling between the troposphere, radiation belts and the middle atmosphere provides information about radiation belt losses, while at the same time leading to new understanding on energy inputs into the middle atmosphere/lower ionosphere. In our previous studies, we considered the lifetimes of radiation belt electrons due to WEP losses, based on Trimpi measurements and an estimate of the a typical WEP burst mean precipitation energy flux (e.g. Rodger and Clilverd, 2002). In this paper we test the reliability of those rates and the validity of the conclusions drawn from those studies by examining a new set of Trimpi observations from New Zealand. Using lightning current distributions we re-consider the expected variation in WEP burst mean precipitation energy so as to more accurately determine the characteristics of "typical" bursts, an important parameter in previous studies (e.g. Burgess and Inan, 1993). Finally, we consider how the rate of energy deposited into the atmosphere by WEP bursts will behave globally, indicating the regional variation in precipitation as an energy input to the middle-atmosphere.

\section{Lightning driven WEP rates}

Earlier studies have used ground based whistler occurrence rate observations as a proxy for Whistler-induced Electron Precipitation (WEP) activity leading to energetic electron precipitation from the radiation belts (e.g. Burgess and Inan, 1993; Abel and Thorne., 1998). This approach may not be particularly reliable, given the suggestion that nonducted whistlers, which are unlikely to be observed on the ground in the conjugate hemisphere, may play an important role (Lauben et al., 1999). We instead follow the recent approach of using Trimpi observations as a more direct proxy for WEP activity leading to ionospheric modifications (e.g. Rodger et al., 2003), combined with global lightning observations.

\subsection{Global observations of lightning activity}

Satellite observations now allow some confidence in the average geographical distribution of total lightning activity and global flash rate. Five years of Optical Transient Detector (OTD) observations have been combined to produce lightning density distributions averaged over the year (Christian et al., 2003), which show strong seasonal variations driven by the different amounts of land in the Northern and Southern Hemispheres. We have previously argued that the global average WEP rate should be directly dependent on the distribution of lightning activity (Rodger and Clilverd, 2002; Rodger et al., 2003), and its seasonal variations (Rodger et al., 2004a). The effect of a given lightning discharge on the radiation belts will be influenced by geomagnetic latitude. In particular, the very strong lightning activity in equatorial 


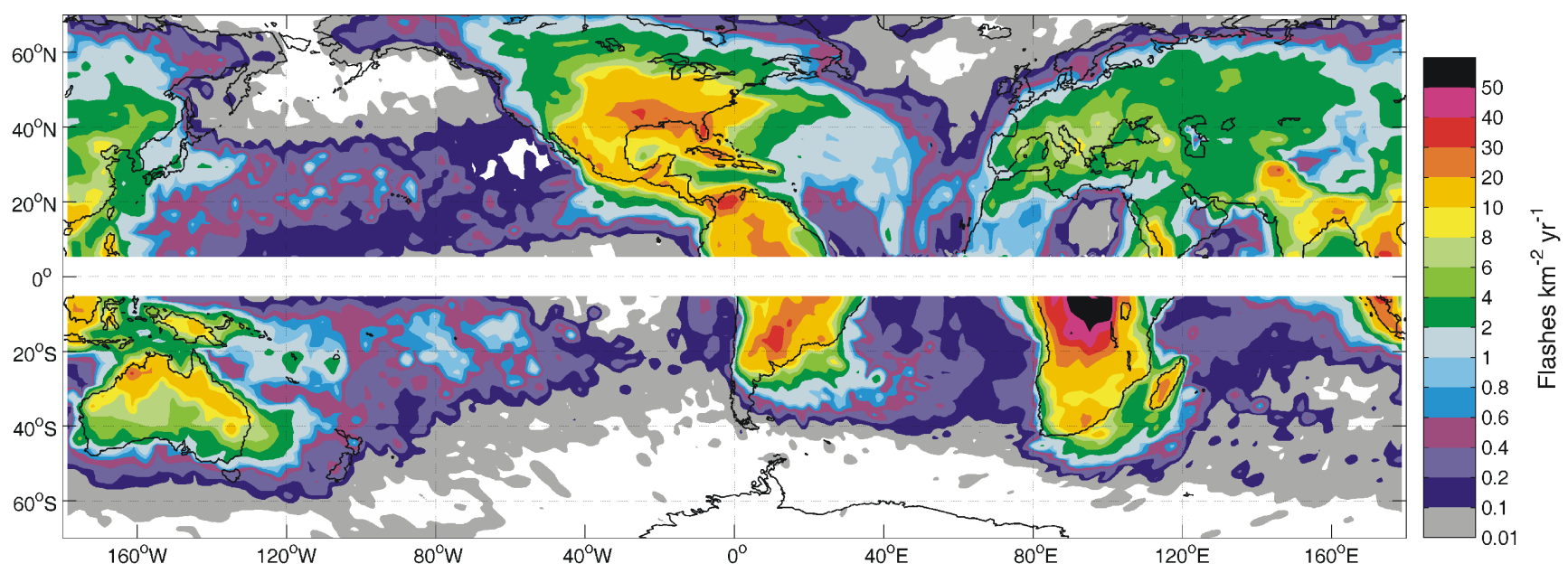

Fig. 1. The annual average global total lightning activity (in units of flashes $\mathrm{km}^{-2} \mathrm{yr}^{-1}$ ) transformed into CGM geomagnetic co-ordinates.

regions is unlikely to influence any part of the radiation belts. For this reason we have transformed the global geographical maps of total lightning activity (in units of flashes $\mathrm{km}^{-2} \mathrm{yr}^{-1}$ ) taken from the OTD Low Resolution Full Climatology dataset (Christian et al., 2003, Fig. 4) into magnetic coordinates. Figure 1 shows the annual average global total lightning activity in Corrected GeoMagnetic (CGM) coordinates based on the Definite/International Geomagnetic Reference Field (DGRF/IGRF) for 2003 at $100 \mathrm{~km}$ altitude, using the GEOPACK software routines. To aid the eye, this figure shows the geographical coastlines also translated into CGM coordinates. Lightning activity has been suppressed on this plot for very low latitudes $\left(<5^{\circ}\right)$, where the GEOPACK calculation is not reliable. As will be seen later, this is unlikely to influence any of the following conclusions. Note that Fig. 1 is an improved geomagnetic coordinate transformation compared with the one presented by Rodger et al. (2004a), which was based on the simpler eccentric geomagnetic dipole field model. This updated map has additional relevance due to the recent observation that plasmaspheric hiss is primarily generated by lightning (Green et al., 2005), based on comparisons between spatial and seasonal occurrence.

\subsection{Average trimpi rates in the Antarctic Peninsula}

We previously estimated global WEP rates and their significance to the radiation belts by considering Trimpi observations at Faraday, Antarctica $\left(65.25^{\circ} \mathrm{S}, 64.27^{\circ} \mathrm{W}, L=2.45\right)$ made over 115 days from 1993 and 1994 (Rodger et al., 2002). As Trimpi are not observed for daytime ionospheric conditions, only days in March through to September were included, avoiding the Antarctic summer. Subionospheric signals from the VLF transmitter NPM $(23.4 \mathrm{kHz}$, Oahu, Hawaii) were recorded at Faraday (Fig. 2, top panel) on an OMSK receiver (Dowden et al., 1994) which logged the phase and amplitude of the signals with a time resolution of $0.4 \mathrm{~s}$. Trimpi events observed were analyzed using a

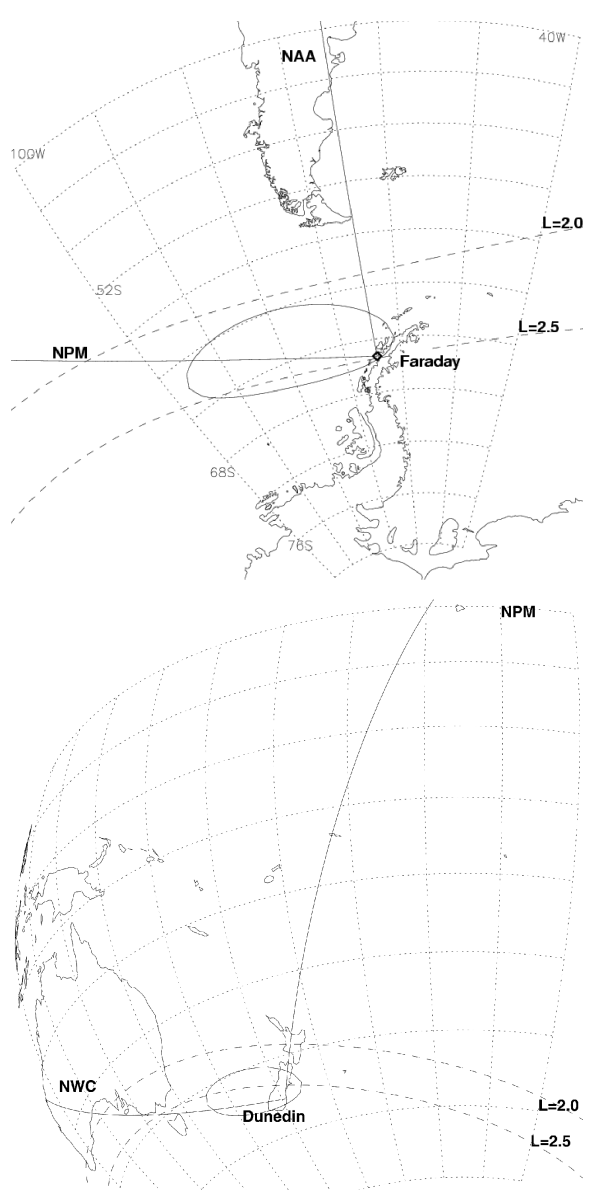

Fig. 2. Maps showing the VLF paths for Trimpi observations undertaken in this study, along with the $L=2.0$ and $L=2.5$ contours at $100 \mathrm{~km}$ altitude. An ellipse marks the WEP modified ionospheric region used in our calculations. Top Panel: the region around the Antarctic Peninsula and the location of Faraday Station. Bottom Panel: the region around New Zealand and the location of Dunedin. 


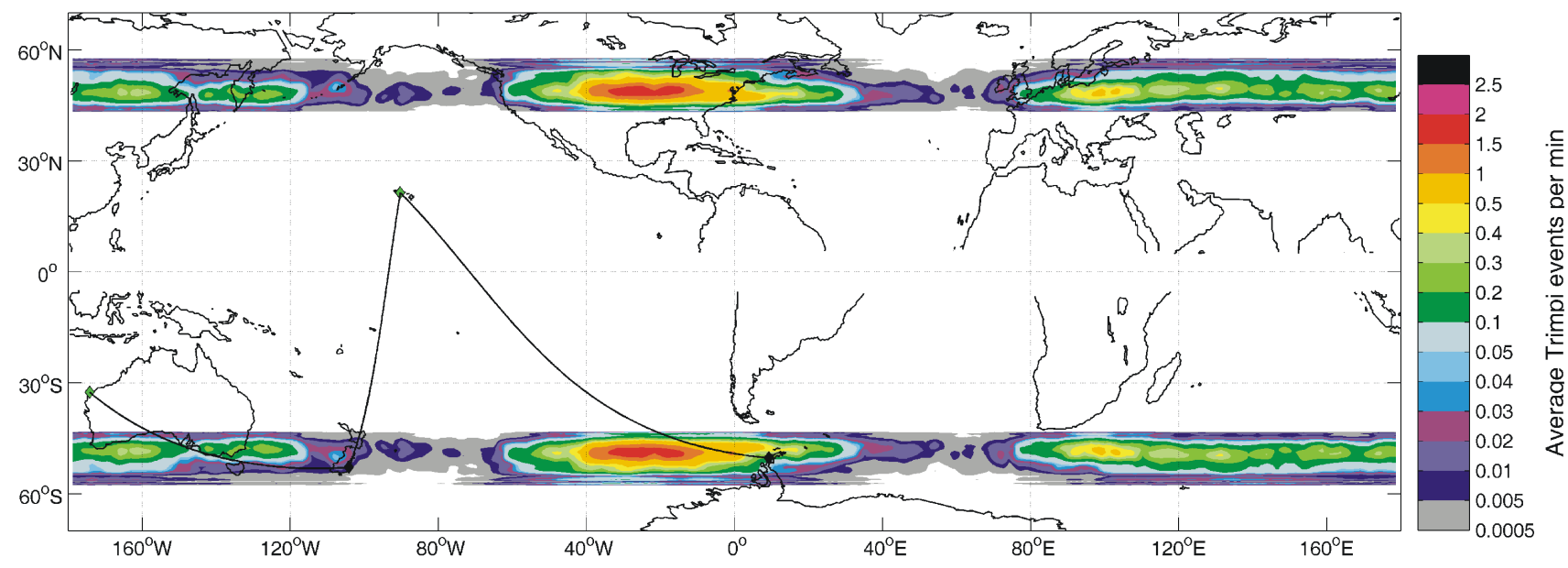

Fig. 3. Map showing the expected global distribution in the rate of WEP producing Trimpi in CGM coordinates. The Great Circle Paths from the transmitters (green diamonds) to the receivers (black diamonds) considered in our study are also shown.

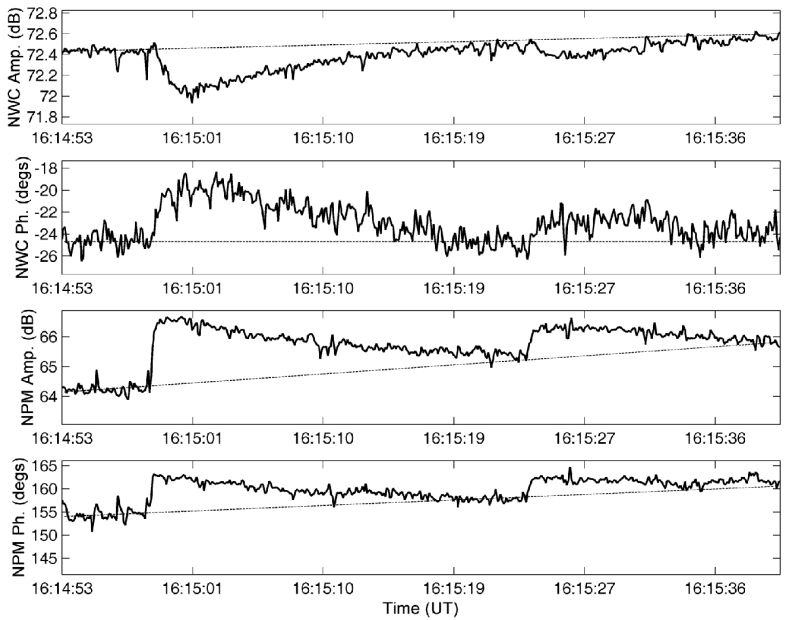

Fig. 4. An example of a fairly large Trimpi event observed on transmissions from NWC and NPM at Dunedin on 1 November 2003. The time resolution is $0.4 \mathrm{~s}$. The unperturbed behaviour of the signals is indicated by the long dashed lines.

simple program to select clear Trimpi events (The use of this program is discussed in more detail elsewhere (Clilverd et al., 2002)). In this time window the most likely rate was one Trimpi perturbation per min, while on $\sim 30 \%$ of days the rate was greater than $2 \mathrm{~min}^{-1}$, and on only $10 \%$ of the days was the rate higher than $3 \mathrm{~min}^{-1}$ (Rodger et al., 2002). Trimpi perturbations observed in the Antarctic Peninsula are known to be strongly associated with high-current cloud to ground lightning occurring around $34^{\circ} \mathrm{N}, 76^{\circ} \mathrm{W}$ (Clilverd et al., 2002, 2004), close to the footprint of $L \approx 2$ flux tubes. From these observations, combined with the seasonality of lightning activity in the Faraday Trimpi producing region, we argued that the representative rate for mean WEP activity affecting the ionosphere observed from Faraday at all times throughout the year would be $0.79 \mathrm{WEP} / \mathrm{min}$ (Rodger et al., 2004a). This mean rate was found after considering the seasonal and diurnal nature of the thunderstorm source, as discussed in that study. The WEP is taken to produce an ellipse shaped ionospheric modification with dimensions $850 \times 2150 \mathrm{~km}$, centred $800 \mathrm{~km}$ away from Faraday on the 12.3 Mm NPM-Faraday Great Circle Path (GCP), the ellipse having its long axis orientated in the magnetic $\mathrm{E} / \mathrm{W}$ direction as shown in Fig. 2. The WEP-modified ionospheric modification is termed a Lightning Induced Enhancement (LIE) "patch". This position and ionospheric modification size are taken from Clilverd et al. (2002), having been determined by observations at multiple Antarctic Peninsula stations.

\subsection{Global distribution of trimpi rate}

On the basis of the Faraday representative Trimpi rate, we may consider the global distribution in WEP producing Trimpi, assuming that the WEP rate is directly linked to lightning activity around the base of the field line which will produce the Faraday LIE oval (Fig. 2, top panel). This assumption seems reasonable, given that SAMPEX and UARS satellite data have revealed hundreds of cases where enhanced electron precipitation losses were associated with individual thunderstorms (Blake et al., 2001). As WEP originates from around the geomagnetic equator through the interaction of a whistler-wave with counter-streaming energetic electrons, one might expect the LIE to be produced only in the source lightning hemisphere. Given the very low lightning levels in Antarctica ( $\sim 4000$ times lower than the conjugate region), this would suggest extremely low Trimpi levels, rather than the high rates experimentally observed. In fact, the magnitude of the Faraday observed Trimpi perturbations have been shown to directly relate to the magnitude of the lightning return stroke peak currents (Clilverd et al., 2004). This is explained by in situ satellite observations of WEP which indicate that a portion of the precipitated electrons backscatter from the atmosphere and bounce repeatedly between the 
northern and Southern Hemispheres, leading to a series of decreasing WEP pulses into both hemispheres over a period of $\sim 2-3$ s (Voss et al., 1998).

Clilverd et al. (2002) showed that as the centre of the LIE patch moves to more than $2 \mathrm{Mm}$ from the receiver the resultant Trimpi perturbations become undetectable for typical noise levels. Therefore only WEP local to the receiver will lead to Trimpi, and not that occurring anywhere else on the transmitter-receiver GCP. Thus the Faraday Trimpi rate should be linked to the lightning activity that occurs near the base of the field lines which produce the Faraday LIE oval, in both the source and conjugate hemisphere. In this manner we have used lightning occurrence rates at Faraday $L$-shells to produce an expected longitudinally varying WEP producing Trimpi rate. This coupling will not, however, be constant with $L$. In situ observations indicate that WEP is rare at low $L$-shells (Voss et al., 1998), despite high lightning activity (Fig. 1), due to increasingly unfavourable gyroresonance conditions (Friedel and Hughes, 1992). This $L$-variation in this coupling can be reasonably estimated from SEEP measurements of how experimentally observed WEP varies with $L$ (Fig. 11, Voss et al., 1998), and thus allow us to estimate the expected global distribution in the rate of WEP producing Trimpi, as shown in Fig. 3. This plot is in CGM coordinates, and includes both "primary" WEP (from lightning in the same hemisphere) and "secondary" WEP (from conjugate lightning), producing high WEP rates due to North American lightning. The GCP from NPM to Faraday is marked on the Figure, as are the paths to our receiver in New Zealand (discussed in Sect. 4). Note that Fig. 3 shows no WEP activity below $L=1.9$ and above $L=3.5$ due to the limitations of the coupling parameters derived from SEEP satellite measurements. For low $L$-shells this is probably a reasonable approximation, as while lightning levels are higher, Trimpi studies indicate very low WEP rates. VLF perturbation observations undertaken in July 1991 from Durban, South Africa, found that the Trimpi rate along an approximately $L=1.7$ east-west path was only $\sim 2.5 /$ day (Friedel et al., 1993). In contrast, at high $L$-values lightning levels are very low, and thus WEP should also be uncommon in this region. It should be noted that strictly speaking, the range of $L$-shells over which WEP is taken to occur $(1.9 \leq L \leq 3.5)$ spans the radiation belt, from the outer part of the inner belt, through the slot region, and including the inner part of the outer belt.

The WEP producing Trimpi rates shown in Fig. 3 should be indicative of the Trimpi rates one would expect to observe at a given location, produced by precipitation local to the receiver. However, one must take care in making this comparison, as the observations of Trimpi require nighttime ionospheric conditions near the receiver end, while Fig. 3 describes the typical mean WEP rate determined from Trimpi observations, once the diurnal and seasonal variations have been taken into account (e.g. Sect. 3.4). While Fig. 3 describes a "Trimpi rate", one might think of this plot as presenting the rate of WEP bursts which could produce an observable Trimpi given a nighttime ionosphere and low noise levels. It is argued below that the true WEP rate will be considerably higher, including the large number of WEP bursts which are too weak to produce observable Trimpi perturbation in the presence of realistic noise levels.

WEP producing Trimpi rates derived in a similar manner to those presented in Fig. 3 have been used to determine the lifetime of energetic electrons in the inner radiation belts for a limited number of selected $L$-shells (Rodger et al., 2003, 2004a). Figure 3 shows that global WEP is strongly dominated by the high mid-latitude lightning activity which occurs in North America, and hence that WEP driven losses from the radiation belt are strongly affected by North American lightning levels. Clearly this indicates that global inner radiation belt fluxes could be potentially influenced by regional climate change occurring in North America, changing local lightning activity levels (Price and Rind, 1994; Leung et al., 2004).

The WEP producing Trimpi rates in Fig. 3, and the earlier lifetime estimates, strongly depend on the Faraday observed Trimpi rate. In order to test the reliability of this estimate, we consider the experimentally observed Trimpi rate from our VLF observations undertaken in New Zealand. From Fig. 3, one can clearly see that the expected Trimpi rate observed from New Zealand will be considerably lower than that reported from Faraday. Indeed, it is likely that our New Zealand observations represent an extreme low in terms of practical WEP observations using Trimpi.

\section{Trimpi observations from New Zealand}

\subsection{Experimental setup}

The lower panel of Fig. 2 shows the VLF radio paths to Dunedin, New Zealand $\left(45.78^{\circ} \mathrm{S}, 170.47^{\circ} \mathrm{E}, L=2.75\right)$ from the US Navy transmitters NPM and NWC $(19.8 \mathrm{kHz}$, North West Cape, Australia). Superimposed on these Great Circle Paths (GCPs) is a WEP produced LIE of similar dimensions to that shown in the Faraday plot. The transmitted amplitude and phase from NPM and NWC were recorded with $0.2 \mathrm{~s}$ resolution using an AbsPAL receiver which is similar to OmniPAL VLF data loggers (Dowden et al., 1998) but modified so that it locks to the GPS generated $1 \mathrm{~s}$ pulse (Bahr et al., 2000), providing "absolute" phase measurements. We examine AbsPAL recordings made from 17 April 2003 through to 26 June 2004. Taking into account NWC and NPM offtimes (we have observations of each transmitter for $\sim 72 \%$ of this time window), there were about $10000 \mathrm{~h}$ of subionospheric measurements taken of both transmitters simultaneously throughout this time period.

\subsection{Dunedin trimpi observations}

Figure 4 shows an example of a fairly large WEP produced Trimpi observed on NWC and NPM in Dunedin on 1 November 2003. Significant changes in amplitude and phase occur at the beginning of the event and relax over tens of seconds. As perturbations are observed with a near-simultaneous onset on both transmitters, we can be confident that the LIE 
Table 1. Calculated scatter amplitude, scatter phase and absolute Trimpi size (amplitude and phase) calculated for the LIE shown in the lower panel of Fig. 2, on transmissions received at Dunedin.

\begin{tabular}{lllll}
\hline NWC & \multicolumn{3}{c}{ NPM } \\
\hline & 1 January & 15 June & 1 January & 15 June \\
Scatt. Amp. & $-14.9 \mathrm{~dB}$ & $-14.4 \mathrm{~dB}$ & $-27 \mathrm{~dB}$ & $-27.5 \mathrm{~dB}$ \\
Scatt. Phase & $155^{\circ}$ & $148^{\circ}$ & $107.5^{\circ}$ & $107^{\circ}$ \\
Trimpi & $-1.5 \mathrm{~dB}, 5.1^{\circ}$ & $-1.48 \mathrm{~dB}, 6.8^{\circ}$ & $-0.11 \mathrm{~dB}, 2.45^{\circ}$ & $-0.1 \mathrm{~dB}, 2.46^{\circ}$ \\
\hline
\end{tabular}

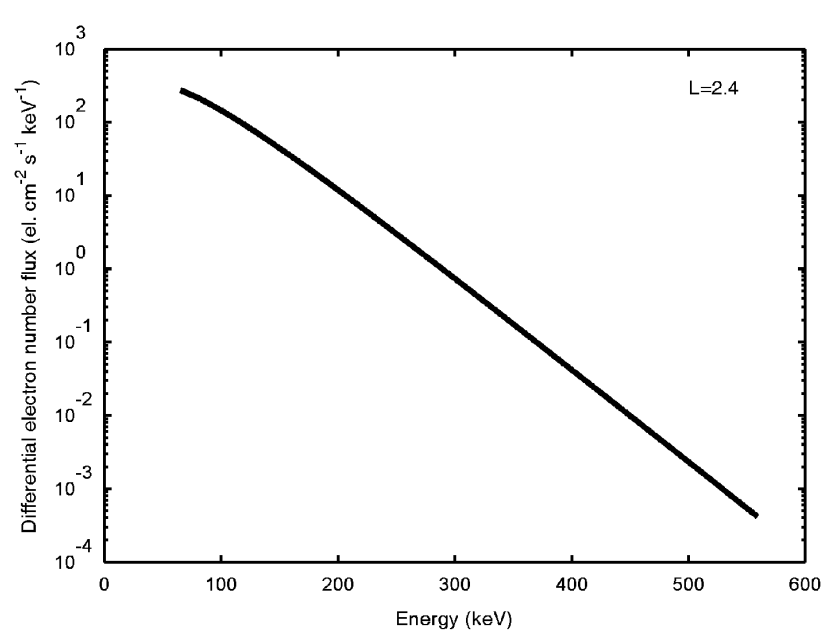

Fig. 5. Differential electron number flux of precipitated electrons in the WEP events used to model Trimpi observations undertaken from Dunedin.

occurred near Dunedin. The unperturbed behaviour of the signals is indicated by the dashed lines. This event had a significance level of 597 on NWC and 1382 on NPM (Clilverd et al., 2002), which are higher than for most of the other events studied here, as this is an example of a large, welldefined, perturbation event.

\subsection{Modelling of Dunedin WEP trimpi}

We model the WEP burst following the approach outlined in Rodger et al. (2003, 2004b). The parameters of the WEP burst have been selected for comparison with the calculations of Faraday Trimpi produced by WEP with a mean precipitation energy flux at $L=2.23$ of $2 \times 10^{-3} \mathrm{ergs} \mathrm{cm}^{-2} \mathrm{~s}^{-1}$ (Rodger et al., 2004b), specifically the "normal" Faraday Trimpi spectral conditions (Clilverd et al., 2004). This modelling assumes an electron beam limited to the range $1-1500 \mathrm{keV}$, produced by a whistler spanning $0.5-5 \mathrm{kHz}$ with power spectral density as given by the lightning spectrum. It is assumed that the WEP lasts $0.2 \mathrm{~s}$, and the cold plasma density at $L=2.23$ is 2536 electrons $/ \mathrm{cm}^{3}$ (Menk et al., 1999). Here the WEP energy spectrum is determined from the spectrum of the trapped electrons in the pitch angle range from the loss cone angle $\left(\alpha_{L C}\right)$ to $\left(\alpha_{L C}+0.5^{\circ}\right)$ given by the empirical AE-5 Inner Zone Electron Model (Teague and Vette, 1972), after Rodger et al. (2003). The spectrum of the WEP burst at $L=2.4$ is shown in Fig. 5. The AE-5 empirical model is used to provide typical "quiet" WEP energy spectra. Intense geomagnetic disturbances can inject energetic electrons into the slot and inner radiation belt, leading to a harder energy spectra for the trapped population. In these cases the WEP energy spectra will also harden, as has been experimentally observed (Inan et al., 1989; Clilverd et al., 2004). As injections into the inner belt are quite rare, we take the "quiet" WEP energy spectra as being more indicative of typical conditions. This WEP burst is used to create a LIE by an application of the expressions in Rees (1989). The resultant Trimpi perturbation was modelled using a linear Born 3-D VLF scattering code (Nunn, 1997), the application of which to LIE is fully described in Rodger et al. (2004b). In order to test the sensitivity to varying neutral atmospheric conditions, the MSIS E-90 model (Hedin, 1991) was run for 1 January and 15 June for $\left(42^{\circ} \mathrm{S}, 167.5^{\circ} \mathrm{E}\right)$. The LIE patch was $800 \mathrm{~km} \times 2000 \mathrm{~km}$, oriented with long axis $10^{\circ}$ from the Dunedin-NWC GCP, and centred at a point $700 \mathrm{~km}$ from Dunedin but displaced $200 \mathrm{~km}$ to the east (lower panel of Fig. 2).

The scattering modelling indicates that the Dunedin observed Trimpi on the transmissions from NWC and NPM will be as outlined in Table 1. Note that the scatter amplitudes and phases in each case are reasonably insensitive to the date taken in the MSIS model. As expected, the relatively small section of the NPM-Dunedin GCP affected by the LIE (lower panel of Fig. 2) leads to small Trimpi perturbations on transmissions from NPM. A perturbation of $0.1 \mathrm{~dB}(\sim-27 \mathrm{~dB}$ scatter amplitude) is similar to the observed NPM-Dunedin evening noise levels, which will strongly influence the number of Trimpi observations. In contrast, the calculated Trimpi due to the same LIE on the NWC-Dunedin path is considerably larger. This is a consequence of the NWC subionospheric signal passing under the long-axis of the LIE (Fig. 2), while NPM passes under the short-axis. Note that the scatter amplitudes and phases for NPM and NWC to Dunedin in Table 1 are comparable to those reported in experimental observations undertaken on the NAA and NPM to Faraday paths (Clilverd et al., 2002; Table 2).

\subsection{Dunedin WEP trimpi rates}

In order to confirm that the WEP occurs locally to Dunedin, i.e. that the LIE is affecting the ionosphere much as shown 
in Fig. 2 (lower panel), we require that Trimpi are observed on transmission from both NWC and NPM at the same time. However, unlike the Antarctic Peninsula, lightning does occur locally to New Zealand. It is well known that there are a number of ionospheric processes which can lead to VLF perturbations (e.g. Rodger, 2003) similar to these WEP produced "classic" Trimpi. Non-WEP perturbations appear to be caused by much faster processes occurring inside and above thunderstorms, e.g. red sprites, elves, and sprite haloes (Rodger, 1999; Haldoupis et al., 2004). We use observations from the New Zealand Lightning Detection Network (NZLDN) to assist us in discriminating between WEP-produced VLF perturbations, and those which occur due to direct lightning processes. The NZLDN is comprised of ten IMPACT ESP2 sensors, deployed to provide optimal detection efficiencies and location accuracies for cloud-toground (CG) return strokes with peak currents $\geq 5 \mathrm{kA}$.

We have examined the entire dataset of Dunedin AbsPAL observations, searching for WEP produced Trimpi occurring simultaneously on both NPM and NWC. This simultaneity is a significant limitation, but one that reduces the influence of non-WEP Trimpi produced by lightning modifications to the ionosphere near the transmitters. Local lightning produced ionospheric modifications (i.e. near the Dunedin receiver) leading to VLF perturbations were discarded from our analysis by considering the NZLDN data along with the observed form of the perturbation (i.e. rise times, phase and amplitude consistency). As a result of this analysis we find 22 WEP produced Trimpi events, which all occur from 00$06 \mathrm{LT}(\mathrm{LT}=\mathrm{UT}+11)$ because of the requirement for nighttime ionospheric conditions on both NPM-Dunedin and NWCDunedin. Inside this time window there were $1600 \mathrm{~h}$ of analyzable observations, leading to a local Dunedin WEP producing Trimpi rate of $2.3 \pm 0.2 \times 10^{-4} \mathrm{~min}^{-1}$. Non-WEP perturbations occurring simultaneously on both paths are about 3 times more common in our data, and will have strongly influenced the conclusions of (Dowden and Adams, 1993), who also considered Dunedin VLF perturbation observations.

The experimentally observed Trimpi rate on NPMFaraday was $0.79 \mathrm{~min}^{-1}$. On the basis of Fig. 3 we expect the WEP producing Trimpi at Dunedin to be $\sim 1 \%$ of that observed at Faraday. However, the Dunedin observed Trimpi rates are 35 times lower than the $\sim 0.008 \mathrm{~min}^{-1}$ expected from considering only the lightning source. This is a consequence of the different location and experimental conditions. The higher $L$-shell of the Dunedin station leads to softer WEP bursts (Vampola and Gorney, 1983), producing smaller Trimpi perturbations, and hence a lower observable WEP producing Trimpi rate for fixed noise conditions. A comparison between Faraday $(L=2.4)$ and Rothera $(L=2.8)$ Trimpi observations shows that this softening will decrease Trimpi rates by a factor of 3 (Clilverd et al., 1999). As the Rothera $L$-shell is very similar to that for Dunedin, this softening factor will be directly applicable in our analysis. The technique used to detect WEP in the Dunedin study is primarily determined by Trimpi occurring on the
NPM to Dunedin path, and hence subionospheric propagation under the semi-minor axis of the LIE, rather than the semi-major axis in the NPM-Faraday case. Experimental observations analyzed as part of our study of simultaneous Trimpi events observed at Faraday on NPM and NAA show that there is typically a factor of 11 times fewer observable events when influenced by propagation under the semiminor axis of the LIE. Taken together, these influences combine to reduce the expected WEP producing Trimpi rate observed at Dunedin by a factor of 33, such that the predicted WEP producing Trimpi rate at Dunedin is $2.4 \times 10^{-4} \mathrm{~min}^{-1}$ $\left(0.79 \times 1 / 3 \times 1 / 11^{1} / 100\right)$. Clearly the predicted and experimentally derived Dunedin WEP producing Trimpi rates are the same. This provides confidence in the global Trimpi rate map shown in Fig. 3, and indeed, the conclusions of our earlier studies (Rodger et al., 2003, 2004a).

\section{Energy precipitated by global WEP activity}

From the expected global variation in the mean rate of WEP producing Trimpi we can estimate the global variation in energy transferred from the Van Allen radiation belts into the atmosphere. A comparison between the relative scattered amplitudes of Faraday-observed Trimpi with the return stroke peak current of the associated lightning discharges found a direct relationship, with stronger lightning producing larger Trimpi due to higher energy fluxes (Clilverd et al., 2004). This study also showed that the relationship changed after geomagnetic disturbances where the trapped electron spectra become harder, such that a given lightning current would produce a larger Trimpi than under undisturbed conditions. Again, we shall take the typical conditions for our consideration. The Clilverd study showed that the magnitude of the WEP mean energy precipitation flux, $F_{W E P}$, is linked to the return stroke peak current of the lightning, $I$ (in $\mathrm{kA}$ ), by the relationship:

$$
F_{W E P}(I)=2 \times 10^{-3}(|I| / 147)^{2.3}\left(\operatorname{ergs~cm}^{-2} \mathrm{~s}^{-1}\right)
$$

Thus, a $147 \mathrm{kA}$ return stroke cloud to ground (CG) lightning flash occurring in the Northern Hemisphere will produce a WEP burst that precipitates into the Southern Hemisphere D-region with mean energy precipitation flux $2 \times 10^{-3} \mathrm{ergs} \mathrm{cm}^{-2} \mathrm{~s}^{-1}$, leading to a Trimpi which is $-20 \mathrm{~dB}$ in scatter amplitude. In the Clilverd et al. (2004) study, Trimpi around $-35 \mathrm{~dB}$ (caused by a $70 \mathrm{kA}$ CG producing a $2 \times 10^{-4} \mathrm{ergs} \mathrm{cm}^{-2} \mathrm{~s}^{-1}$ WEP burst) were at the limit of detection, while the largest Trimpi observed $(-10 \mathrm{~dB}$ scatter amplitude) was associated with a $242 \mathrm{kA}$ flash producing a $6 \times 10^{-3} \mathrm{ergs} \mathrm{cm}^{-2} \mathrm{~s}^{-1}$ WEP burst. We expect that relation (1) will hold for larger and smaller lightning discharges, producing larger and smaller WEP bursts. In fact, this study suggests that many more WEP bursts should be occurring than suggested by observed Trimpi rates, as the majority are caused by lightning with $I<70 \mathrm{kA}$ and hence will be extremely difficult to detect as subionospheric perturbations, 

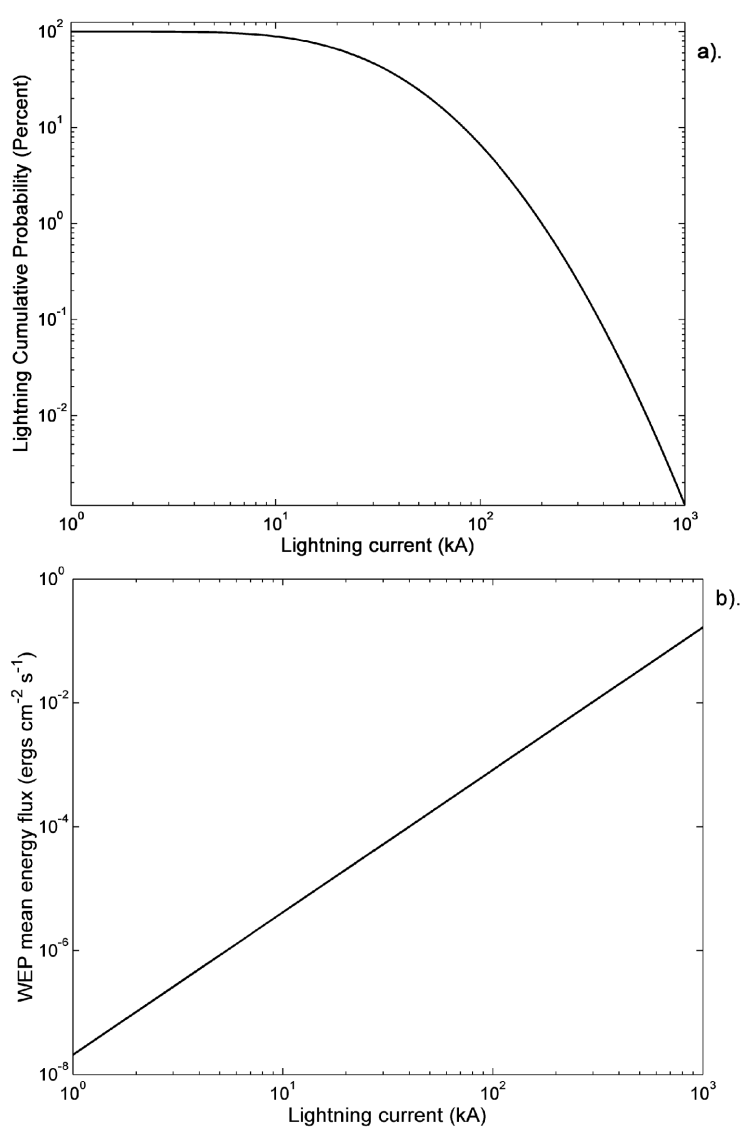

Fig. 6. (Top panel) Cumulative statistical distribution for CG lightning return stroke peak currents. (Lower panel) Magnitude of the WEP mean energy precipitation flux expected from a given lightning return stroke peak current.

even though the bursts will be precipitating energetic electrons into the D-region.

\subsection{Distribution of CG lightning currents}

The distributions of CG lightning return stroke peak currents are described by log-normal distributions. Various authors have determined the cumulative probability distribution for lightning currents, as detailed in Rakov and Uman (Chpt. 4, 2003), and Golde (Chpt. 9, 1977). The study by Popolansky (1972), as referenced by Golde (Chpt. 9, 1977) considered 624 return stroke peak current measurements with a lower cutoff of $2 \mathrm{kA}$, including both negative and positive ground discharges. The measurements indicated that $95 \%$ of lightning had currents greater than $\sim 6-7 \mathrm{kA}$, a median of greater than $28 \mathrm{kA}$, while $1 \%$ had return stroke peak currents $>200 \mathrm{kA}$. From these values we can construct a cumulative frequency distribution for CG lightning return stroke peak currents, as shown in the top panel of Fig. 6.

The WEP mean energy precipitation flux, $F_{W E P}$, expected from the CG lightning return stroke peak currents shown in the top panel of Fig. 6 is shown in the lower panel of this figure. The limit of WEP detection using the Trimpi technique $\left(\sim 2 \times 10^{-4} \mathrm{ergs} \mathrm{cm}^{-2} \mathrm{~s}^{-1}\right.$ WEP burst) occurs for lightning with currents that are considerably higher than the median values, while the non-linear nature of Eq. (1) indicates that high peak current lightning will produce extremely large WEP bursts. On the basis of Fig. 6 only $\sim 14 \%$ of CG lightning would have currents large enough to produce an observable Trimpi in the low-noise conditions of the Antarctic, suggesting that the actual WEP rates due to all CGs (with $>2 \mathrm{kA}$ ) currents are likely to be $\sim 7$ times higher than that determined from the Faraday-observed Trimpi rate (i.e. a mean $\mathrm{WEP}$ activity rate $\sim 6 \mathrm{WEP} / \mathrm{min}$ ), although with very low energy fluxes.

\subsection{Typical WEP burst characteristics}

On the basis of the cumulative statistical distribution for CG lightning shown above, we can determine the mean energy precipitation flux of a typical WEP burst, by considering the WEP fluxes expected from Eq. (1) for CGs with currents from 2-960 kA. As noted above, the lower current range of $2 \mathrm{kA}$ comes from the probability distribution itself, while the upper threshold is less certain. We take $960 \mathrm{kA}$ on the basis of the discussions in Rakov and Uman (Pg. 145-146, 2003), who note that the largest peak current observed by the U.S. National Lightning Detection Network (NLDN) in 14 summer months over 1991-95 was $~ 960$ kA (Lyons et al., 1998). However, the same authors also point out that these CG peak current values come from estimates using an empirical formula applied to the observed peak values in the lightning radiation magnetic field. The empirical formula has only been tested out to $60 \mathrm{kA}$ (Orville, 1999), and it is worth noting that directly measured CG current waveforms found in the literature do not exceed $300 \mathrm{kA}$ or so (Rakov and Uman, 2003).

The WEP burst energy flux precipitating into the Southern Hemisphere D-region in an "average" WEP event, produced by a Northern Hemisphere CG lightning with currents from 2-960 kA, is $F_{W E P}=1.4 \times 10^{-3} \mathrm{ergs} \mathrm{cm}^{-2} \mathrm{~s}^{-1}$. For the case where the upper lightning threshold is $300 \mathrm{kA}$ the mean WEP burst energy flux is $8 \times 10^{-4} \mathrm{ergs} \mathrm{cm}^{-2} \mathrm{~s}^{-1}$. Thus it seems reasonable to suggest that a typical WEP burst impacting the Southern Hemisphere D-region at $L \sim 2.3$ will have mean precipitation energy fluxes of $\sim 1 \times 10^{-3} \mathrm{ergs} \mathrm{cm}^{-2} \mathrm{~s}^{-1}$.

\subsection{Earlier estimates of typical WEP}

These values are smaller than the "typical" $0.2 \mathrm{~s}$ WEP burst with mean precipitation energy flux of $2 \times 10^{-3} \mathrm{ergs} \mathrm{cm}^{-2} \mathrm{~s}^{-1}$, used in radiation belt loss rate calculations (e.g. Rodger et al., 2003) on the basis of satellite WEP observations (Voss et al., 1998) and ground measurements of whistlers (Burgess and Inan, 1993). However, the radiation belt loss rate calculations described in these earlier studies relied on a WEP rate determined by Trimpi observations, and hence appropriate for CGs with currents $>70 \mathrm{kA}$. For lightning in the range 70 $300 \mathrm{kA}$ the mean WEP precipitation energy flux burst is $2 \times 10^{-3} \mathrm{ergs} \mathrm{cm}^{-2} \mathrm{~s}^{-1}$, while it is $\sim 3 \times 10^{-3} \mathrm{ergs} \mathrm{cm}^{-2} \mathrm{~s}^{-1}$ 


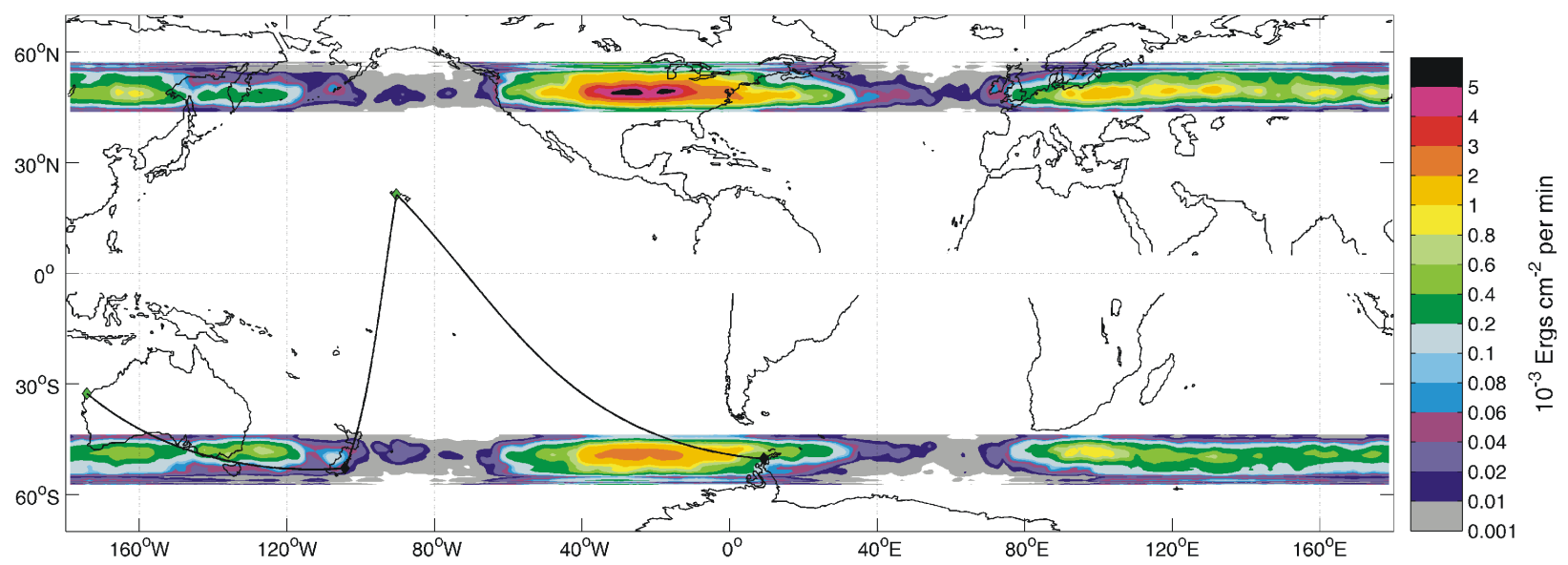

Fig. 7. Map showing the expected global distribution in the rate of energy deposited by WEP into the atmosphere, in the same form as Fig. 3.

for WEP due to lightning ranging over 70-960 kA, much like that used previously. The WEP producing Trimpi rates (e.g. $0.79 \mathrm{~min}^{-1}$ at Faraday) applied in those studies will be valid for the WEP energy fluxes of $\sim 2-3 \times 10^{-3} \mathrm{ergs} \mathrm{cm}^{-2} \mathrm{~s}^{-1}$, produced by lightning that will lead to Trimpi perturbations. Thus it appears that the conclusions of these studies (Rodger et al., 2003, 2004a) concerning the importance of WEP as a inner radiation belt loss process are still valid despite our improved understanding of troposphere to magnetosphere coupling.

\subsection{Global WEP activity estimates}

Taking a typical WEP burst impacting the Southern Hemisphere D-region at $L=2.23$ as having a mean precipitation energy flux of $\sim 1 \times 10^{-3} \mathrm{ergs} \mathrm{cm}^{-2} \mathrm{~s}^{-1}$, lasting $0.2 \mathrm{~s}$, and driven by all CGs with $>2 \mathrm{kA}$ currents, the Trimpi rates shown in Fig. 3 can be used to determine the total WEP rate, and hence the variation in energy precipitated by global WEP activity, as shown in Fig. 7. Here we assume that the WEP burst precipitated into the atmosphere conjugate to the source lightning flash will be half the magnitude of that precipitated into the source hemisphere. This assumption is based on the in-situ observations of WEP Event D by the SEEP experiment (Fig. 15, Voss et al., 1998), showing that about half as many electrons precipitate into the conjugate (Southern) Hemisphere as precipitate in the lightning-source (Northern) Hemisphere. As a consequence the north-south symmetry seen in Fig. 3 is disrupted, and the rate of energy deposited by WEP into the atmosphere is higher for the Northern Hemisphere than the Southern.

As an example of the calculations shown in Fig. 7, consider the following situation: the peak Trimpi rate to the west of Faraday is $\sim 1.75 \mathrm{~min}^{-1}$, shown as the red region in Fig. 3, which suggests a total mean WEP rate of $\sim 12 \mathrm{~min}^{-1}$ once WEP produced by low-current lightning that will not be observed as Trimpi are allowed for. This region maps to a precipitated energy of $\sim 2.5 \times 10^{-3} \mathrm{ergs} \mathrm{cm}^{-2} \mathrm{~min}^{-1}$ as shown by the orange region in Fig. 7 (1.75 Trimpi $\min ^{-1} \times$
7 WEP Trimpi ${ }^{-1} \times 0.2 \mathrm{~s} \times 1 \times 10^{-3} \mathrm{ergs} \mathrm{cm}^{-2} \mathrm{~s}^{-1}$ ). As the north-south symmetry seen in Fig. 3 is disrupted the rate of energy deposited by WEP into the atmosphere is higher for the Northern Hemisphere than the southern, as expected from the variation in lightning activity (Fig. 1). This results in the mean WEP energy input rate for all longitudes at $L \sim 2.3$ in this Northern Hemisphere being almost twice that for the Southern Hemisphere $\left(8 \times 10^{-4} \mathrm{ergs} \mathrm{cm}^{-2} \mathrm{~min}^{-1}\right.$ c.f. $4.7 \times 10^{-4} \mathrm{ergs} \mathrm{cm}^{-2} \mathrm{~min}^{-1}$ ). The longitudinal variation in lightning activity is so much larger than this factor of 2 that the Antarctic Peninsula region has the second mostsignificant energy input rate, despite being largely devoid of lightning. The strongest energy inputs occur over North America, as expected by the high lightning activity occurring in this region at high geomagnetic latitudes (Rodger et al., Fig. 1, 2004a). Nonetheless, most of the global lightning will not generate WEP, as it occurs in the 3 tropical "chimney" regions of tropical America and Africa, and the Maritime Continent (SE Asia and northern Australia and the Indonesian archipelago) (Christian et al., 2003, Fig. 4).

Because of the coupling between lightning and observed WEP (Sect. 2.3), virtually all of the energy deposited by WEP occurs in the bands shown in Figs. 3 and 7. The mean rate of energy deposited by WEP into the atmosphere inside these bands is $3 \times 10^{-4} \mathrm{ergs} \mathrm{cm}^{-2} \mathrm{~min}^{-1}$, varying from a low of zero to the North American high of $5.6 \times 10^{-3} \mathrm{ergs} \mathrm{cm}^{-2} \mathrm{~min}^{-1}$.

\section{Uncertainties in lightning current distributions}

The Popolansky (1972) cumulative probability distribution for lightning currents we use to determine typical WEP energy precipitation fluxes is based on a relatively limited set of CG lightning current measurements, primarily from strikes on towers, chimneys and lightning rods. While the results compiled into the Popolansky distribution are still used to a large extent as the primary reference source for lightning research (Rakov and Uman, Chpt. 4, 2003), there are 
indications that lightning over land may not have the same current distributions as lightning which occurs over water. An examination of ten years of NLDN data showed significant variation in the mean return-stroke currents across the continental U.S. (Orville and Huffines, 2001). In particular, this study showed a sharply defined increase in the median peak currents of negative CGs at the land/salt water boundaries, but interestingly, not over the Great Lakes. While initially considering that this might be due to propagation effects or detector sensitivities, no similar effect was seen for positive CGs in the same dataset. More dramatically, Lyons et al. (1998) noted that negative Large Peak Current CGs (LPCCGs) of $>200 \mathrm{kA}$ were far more common over the Gulf of Mexico and Atlantic than the adjacent land areas, an effect not noted in positive LPCCG distributions in the same areas. As noted by Orville and Huffines (2001), "At the moment, we have no explanation for this observation." Presently, there is no accepted physical mechanism to explain these observations. The sharply-bounded changes in negative CG return-stroke peak currents along the U.S. Gulf and Atlantic coastlines may reflect either a network anomaly or a physical process connected with highly conductive salt water. This would tend to increase the precipitation energy fluxes for WEP bursts produced by lightning that occurs over sea-water, and hence the observable Trimpi rate. We note that this may be the case for the Faraday observations, and hence further testing of the WEP rates used in our study is warranted.

There is also the question of whether intracloud (IC) lightning flashes (sometimes simply termed "cloud flashes") can generate WEP pulses. It has generally been assumed that CGs are the dominant sources of VLF sferics, and hence whistlers. However, recent studies comparing MF band (0.3$3 \mathrm{MHz}$ ) commercial lightning detection networks with VLF lightning observations indicate that a significant fraction, on the order of $50 \%$, of sferics detected several Mm from their sources are produced by ICs. An experimental VLF lightning location network operated in the US detected $\sim 65 \%$ of CG discharges and $\sim 13 \%$ of IC discharges (Morales et al., $2005^{1}$ ), such that roughly half the events detected were due to IC discharges. This is roughly consistent with a comparison undertaken in Australia between commercial and experimental VLF World Wide Lightning Location network data. In that case the unmatched event set, assumed to be produced by cloud flashes, was $\sim 130 \%$ of the size of the matched set of CG discharges (Rodger et al., 2005a). VLF observations of sferics made at Palmer station, Antarctica believed to be from a storm in the southern USA have been contrasted with CG observations made by NLDN (Wood and Inan, 2002), leading the authors to conclude that both ICs and CGs were present in the Palmer sferic data. It seems reasonable to

\footnotetext{
${ }^{1}$ Morales, C. A., Weiman, J. A., Anagnostou, E. N., Goodman, S., and Williams, E.: Continuous long-range thunderstorm monitoring by a VLF receiver network: Location error analysis and cloudto-ground and intra-cloud detection efficiency, J. Atmos. Ocean. Tech., submitted, 2005.
}

expect the sferics produced by these strong cloud flashes could lead to whistlers, and hence to WEP. An attempt to link whistlers observed by the MAGION 5 low-altitude satellite with ground-based commercial CG lightning detections found few agreements, possibly because of cloud-flash produced whistlers (J. Chum, 2004, personal communication).

\section{Summary}

In this paper we have examined WEP burst characteristics in order to determine the global distribution of energy deposited into the middle atmosphere from whistler-induced electron precipitation. To do this we have made use of an improved global geomagnetic conjugate mapping tool based use of the DGRF/IGRF standard magnetic field model. As a result of this tool a prediction has been made of the global distribution of WEP bursts, as well as locally produced Trimpi perturbations. Future ground-based studies into WEP should be guided by these findings. Previous studies using lightningdriven precipitation burst rates have estimated losses from the inner radiation belts. In order to confirm the reliability of those rates and the validity of the conclusions drawn from those studies, we have analyzed a new set of New Zealand Trimpi data to test our global understanding of troposphere to magnetosphere coupling. The Trimpi rates observed in New Zealand data are consistent with those predicted from the global variation in the lightning sources, once the different experimental configurations are taken into account. This provides confidence in the global WEP rate map presented here and indeed, the conclusions of our earlier studies (Rodger et al., 2003, 2004a).

On the basis of CG lightning current distributions, we determined the mean energy precipitation flux of typical WEP bursts. For WEP that produce observable Trimpi perturbations, mean precipitation energy fluxes will be 2$3 \times 10^{-3} \mathrm{ergs} \mathrm{cm}^{-2} \mathrm{~s}^{-1}$, as they are caused by lightning with larger currents. Taking into account the full range of lightning currents, a typical WEP burst at $L \sim 2.3$ will have a mean precipitation energy flux of $\sim 1 \times 10^{-3} \mathrm{ergs} \mathrm{cm}^{-2} \mathrm{~s}^{-1}$. The precipitation of energetic electrons by these WEP bursts will lead to a mean rate of energy deposited into the atmosphere from $L=1.9-3.5$ of $3 \times 10^{-4} \mathrm{ergs} \mathrm{cm}^{-2} \mathrm{~min}^{-1}$, spatially varying from a low of zero above some ocean regions to highs of $\sim 3-6 \times 10^{-3} \mathrm{ergs} \mathrm{cm}^{-2} \mathrm{~min}^{-1}$ above North America and its conjugate region.

Acknowledgements. C. J. Rodger would like to thank T. Husheer of Dunedin for his support. He is grateful to the New Zealand MetService Ltd. for collecting the NZLDN data, most particularly P. Fisher and J. Glover for helping him to understand its operation. This research was partially supported by the New Zealand Marsden Research Fund contract 02-UOO-106. The authors would like to thank S. Werner for preparing the TrimDet software, as well as the NASA LIS/OTD Science Team and Global Hydrology and Climate Center (GHCC) for collecting and providing the satellite observed lightning data.

Topical Editor U.-P. Hoppe thanks J. Green and another referee for their help in evaluating this paper. 


\section{References}

Abel, B. and Thorne, R. M.: Electron scattering loss in earth's inner magnetosphere-2. Sensitivity to model parameters, J. Geophys. Res., 103, 2397-2407, 1998.

Barr, R., Jones, D. Ll., and Rodger, C. J.: ELF and VLF Radio Waves, J. Atmos. Sol. Terr. Phys., 62(17-18), 1689-1718, 2000.

Bahr, J. L., Brundell, J. B., Hardman, S. F., and Dowden, R. L.: Multi-instrument coincident detection of sprites, Phys. Chem. Earth (B), 25, 417-422, 2000.

Blake, J. B., Inan, U. S., Walt, M., Bell, T. F., Bortnik, J., Chenette, D. L., and Christian, H. J.: Lightning-induced energetic electron flux enhancements in the drift loss cone, J. Geophys. Res., 106, 29733-29744, 2001.

Burgess, W. C. and Inan, U. S.: The role of ducted whistlers in the precipitation loss and equilibrium flux of radiation belt electrons, J. Geophys. Res., 98, 15 643-15 665, 1993.

Carpenter, D. L. and LaBelle, J. W.: A study of whistlers correlated with bursts of electron precipitation near $\mathrm{L}=2$, J. Geophys. Res., 87, 4427-4434, 1982.

Carpenter, D. L. and Orville, R. E.: The excitation of active whistler mode signal paths in the magnetosphere by lightning -2 case studies, J. Geophys. Res., 94, 8886-8894, 1989.

Christian, H. J., Blakeslee, R. J., Boccippio, D. J., Boeck, W. L., Buechler, D. E., Driscoll, K. T., Goodman, S. J., Hall, J. M., Koshak, W. J., Mach, D. M., and Stewart, M. F.: Global frequency and distribution of lightning as observed from space by the Optical Transient Detector, J. Geophys. Res., 108(D1), doi:10.1029/2002JD002347, 2003.

Clilverd, M. A., Yeo, R. F., Nunn, D., and Smith, A. J.: Latitudinally dependent Trimpi effects: Modeling and observations, J. Geophys. Res., 104, 19881-19887, 1999.

Clilverd, M. A., Nunn, D., Lev-Tov, S. J., Inan, U. S., Dowden, R. L., Rodger, C. J., and Smith, A. J.: Determining the size of lightning-induced electron precipitation patches, J. Geophys. Res., 107(A8), doi:10.1029/2001JA000301, 2002.

Clilverd, M. A., Rodger, C. J., and Nunn, D.: Radiation belt electron precipitation fluxes associated with lightning, J. Geophys. Res., 109(12), A12208, doi:10.1029/2004JA010644, 2004.

Dowden, R. L. and Adams, C. D. D.: Size and location of lightninginduced enhancements from measurement of VLF phase and amplitude perturbations on multiple antennas, J. Atmos. Terr. Phys., 55, 1335-1359, 1993.

Dowden, R. L., Adams, C. D. D., Brundell, J., and Dowden, P. E.: Rapid onset, rapid decay (RORD), phase and amplitude perturbations of VLF subionospheric transmissions, J. Atmos. Terr. Phys., 56, 1513-1527, 1994.

Dowden, R. L., Hardman, S. F., Rodger, C. J., and Brundell, J. B.: Logarithmic decay and Doppler shift of plasma associated with sprites, J. Atmos. Sol. Terr. Phys., 60, 741-753, 1998.

Dungey, J. W.: Loss of Van Allen electrons due to whistlers, Planet. Space Sci., 11, 591-595, 1963.

Friedel R. H. W. and Hughes, A. R. W.: Trimpi events on low latitude paths - an investigation of gyroresonance interactions at low L-values, J. Atmos. Terr. Phys., 54, 1375-1386, 1992.

Friedel R. H. W., Hughes, A. R. W., Dowden, R. L., and Adams, C. D. D.: First observations of Trimpi events at Durban ( $\mathrm{L}=1.69)$ using an OMSKI receiver, J. Geophys. Res., 98, 1571-1580, 1993.

Golde, R. H.: Lightning currents and related parameters, in: Lightning, vol. 1, edited by: Golde, R. H., 309-350, Academic Press, New York, 1977.
Green, J. L., Boardsen, S., Garcia, L., Taylor, W. W. L., Fung, S. F., and Reinisch, B. W.: On the origin of whistler mode radiation in the plasmasphere, J. Geophys. Res., 110, A03201, doi:10.1029/2004JA010495, 2005.

Haldoupis, C., Neubert, T., Inan, U. S., Mika, A., Allin, T. H., and Marshall, R. A.: Subionospheric early VLF signal perturbations observed in one-to-one association with sprites, J. Geophys. Res., 109(A10303), doi:10.1029/2004JA010651, 2004.

Hedin, A. E.: Extension of the MSIS thermospheric model into the middle and lower atmosphere, J. Geophys. Res., 96, 1159-1172, 1991.

Helliwell, R. A., Katsufrakis, J. P., and Trimpi, M. L.: Whistlerinduced amplitude perturbation in VLF propagation, J. Geophys. Res., 78, 4679-4688, 1973.

Inan, U. S., Walt, M., Voss, H. D., and Imhof, W. L.: Energy spectra and pitch angle distributions of lightning-induced electron precipitation: Analysis of an event observed on the S81-1 (SEEP) satellite, J. Geophys. Res., 94, 1379-1401, 1989.

Kennel, C. F. and Petschek, H. E.: Limit on stably trapped particle fluxes, J. Geophys. Res., 71(1), 1-27, 1966.

Lauben, D. S., Inan, U. S., and Bell, T. F.: Subionospheric VLF signatures of oblique (nonducted) whistler-induced precipitation, Geophys. Res. Lett., 26, 2633-2636, 1999.

Leung, L. R., Qian, Y., Bian, X. D., Washington, W. M., Han, J. G., and Roads, J. O.: Mid-century ensemble regional climate change scenarios for the western United States, Climatic Change, 62(13), 75-113, 2004.

Lyons, W. A., Uliasz, M., and Nelson, T. E.: Large peak current cloud-to-ground lightning flashes during the summer months in the contiguous United States, Mon. Wea. Rev., 126, 2217-2233, 1998.

Meredith, N. P., Horne, R. B., Iles, R. H. A., Thorne, R. M., Heynderickx, D., and Anderson, R. R.: Outer zone relativistic electron acceleration associated with substorm-enhanced whistler mode chorus, J. Geophys. Res., 108, 1016, 2002.

Menk, F. W., Orr, D., Clilverd, M. A., Smith, A. J., Waters, C. L., Milling, D. K., and Fraser, B. J.: Monitoring spatial and temporal variations in the dayside plasmasphere using geomagnetic field line resonances, J. Geophys. Res., 104(A9), 19 955-19969, 1999.

Nunn, D.: On the numerical modelling of the VLF Trimpi effect, J Atmos. Sol. Terr. Phys., 59, 537-560, 1997.

Orville, R. E.: Comments on "Large peak current cloud-to-ground lightning flashes during the summer months in the contiguous United States", Monthly Weather Rev., 127, 1937-1938, 1999.

Orville, R. E. and Huffines, G. R.: Cloud-to-Ground lightning in the United States: NLDN results in the first decade, 1989-98, Mon. Wea. Rev., 129, 1179-1193, 2001.

Pasko, V. P. and Inan, U. S.: Recovery signatures of lightningassociated VLF perturbations as a measure of the lower ionosphere, J. Geophys. Res., 99, 17 523-17 537, 1994.

Popolansky, F.: Frequency distribution of amplitudes of lightning currents, Electra., 22, 139-146, 1972.

Price, C. and Rind, D.: Possible Implications Of Global ClimateChange On Global Lightning Distributions And Frequencies, J. Geophys. Res., 99 (D5), 10 823-10 831, 1994.

Rakov, V. A. and Uman, M. A.: Lightning; Physics and Effects, Cambridge University Press, Cambridge, 2003.

Rees, M. H.: Physics and chemistry of the upper atmosphere, Cambridge University Press, Cambridge, 1989.

Rodger, C. J.: Red sprites, upward lightning and VLF perturbations, Rev. Geophys., 37, 317-336, 1999. 
Rodger, C. J.: Subionospheric VLF perturbations associated with lightning discharges, J. Atmos. Sol. Terr. Phys., 65, 591-606, 2003.

Rodger, C. J. and Clilverd, M. A.: Inner radiation belt electron lifetimes due to whistler-induced electron precipitation (WEP) driven losses, Geophys. Res. Lett., 29(19), doi:10.1029/2002GL015795, 2002.

Rodger, C. J., Clilverd, M. A., and McCormick, R. J.: Significance of lightning generated whistlers to inner radiation belt electron lifetimes, J. Geophys. Res., 108(12), 1462, doi:10.1029/2003JA009906, 2003.

Rodger, C. J., McCormick, R. J., and Clilverd, M. A.: Testing the importance of precipitation loss mechanisms in the inner radiation belt, Geophys. Res. Lett., 31(10), L10803, 10.1029/2004GL019501, 2004a.

Rodger, C. J., Nunn, D., and Clilverd, M. A.: Investigating radiation belt losses though numerical modelling of precipitating fluxes, Ann. Geophys., 22(10), 3657-3667, 2004b,

SRef-ID: 1432-0576/ag/2004-22-3657.

Rodger, C. J., Brundell, J. B., and Dowden, R. L.: Location accuracy of long distance VLF lightning location network: Post algorithm upgrade, Ann. Geophys., 23(2), 277-290, 2005a, SRef-ID: 1432-0576/ag/2005-23-277.

Rycroft, M. J.: Enhanced energetic electron intensities at $100 \mathrm{~km}$ altitude and a whistler propagating through the plasmasphere, Planet. Space Sci., 21, 239-251, 1973.
Storey, L. R. O.: An investigation of whistling atmospherics, Phil. Trans. Roy. Soc. (London), 246, 113-117, 1953.

Teague, M. and Vette, J.: The inner zone electron model AE-5, National Space Science Data Center, Rep. NSSDC 72-10, Greenbelt, Md., 1972.

Tsurutani, B. T. and Lakhina, G. S.: Some basic concepts of waveparticle interactions in collisionless plasmas, Rev. Geophys., 35(4), 491-501, 1997.

Van Allen, J. A.: Energetic particles in the Earth's external magnetic field, in Discovery of the Magnetosphere, (Eds.) Gillmor, C. S. and Spreiter, J. R., History of Geophysics, 7, 235-251, American Geophysical Union, Washington, D.C., 1997.

Vampola, A. L. and Gorney, D. J.: Electron energy deposition in the middle atmosphere, J. Geophys. Res., 88, 6267-6274, 1983.

Voss, H. D., Walt, M., Imhof, W. L., Mobilia, J., and Inan, U. S.: Satellite observations of lightning-induced electron precipitation, J. Geophys. Res., 103, 11 725-11 744, 1998.

Walt, M.: Source and loss processes for radiation belt particles, in Radiation Belts: Models and Standards, (Ed.) Lemaire, J. F., Heynderickx, D., and Baker, D. N., Geophysical Monograph, 97, 1-13, American Geophysical Union, Washington, D.C., 1996.

Wood, T. G. and Inan, U. S.: Long-range tracking of thunderstorms using sferic measurements, J. Geophys. Res., 107(21), 4553, doi:10.1029/2001JD002008, 2002. 